\title{
Is there a role for fluorine-18 fluorodeoxyglucose positron emission tomography imaging in medullary thyroid cancer?
}

\author{
Saila Kauhanen • Camilla Schalin-Jäntti
}

Published online: 14 July 2012

(C) Springer Science+Business Media, LLC 2012

Medullary thyroid cancer (MTC) is an uncommon and challenging malignancy. In spite of aggressive primary treatment with total thyroidectomy and modified neck lymph node dissection, approximately $50 \%$ of the patients have persistent or recurrent disease [1,2]. The recent European Thyroid Association guidelines [3] recommend surgery and other local treatment modalities (such as external beam radiation therapy, radiofrequency ablation, and chemoembolization) for the treatment of distant metastases in patients with low tumor burden (distant metastases limited to single organ) and stable disease, while patients with symptoms, significant tumor burden and progressive disease should receive novel targeted systemic therapies. However, imaging of recurrent disease in MTC is difficult and still poses a major problem in the management of the disease.

The serum tumor markers calcitonin $(\mathrm{Ct})$ and carcinoembryonic antigen (CEA) and their doubling times (Dt) are used to assess progression rate and also reflect tumor burden in MTC. However, conventional imaging (CI) is often negative in patients with Ct concentrations $<150 \mathrm{pg} /$ $\mathrm{mL}$ [3]. It is estimated that $\mathrm{CI}$ identifies metastases in about $40 \%$ of patients with biochemical evidence of recurrence. The probability of detecting metastases increases in patients with increasing $\mathrm{Ct}$ and CEA concentrations and shortening Dts. Of note, some patients with poorly

S. Kauhanen $(\bowtie)$

Turku PET Centre and Department of Surgery, Turku University Hospital, Turku, Finland

e-mail: spkauha@utu.fi

C. Schalin-Jäntti

Division of Endocrinology, Department of Medicine, Helsinki University Hospital and University of Helsinki, Helsinki, Finland differentiated and aggressive metastatic MTC have low Ct or discrepantly high CEA concentrations. The recommended CI protocol is extensive and comprises neck ultrasonography for detection of cervical lymph node metastases, computed tomography (CT) of thorax for lung and mediastinal lesions, magnetic resonance imaging (MRI) for liver and bone metastases of the spine and pelvis and bone scintigraphy. Modern whole body imaging with positron emission tomography (PET) and integrated CT could thus represent an alternative to $\mathrm{CI}$, at least for patients with negative $\mathrm{CI}$ imaging.

In this issue of the Endocrine, Treglia et al. [4] report a meta-analysis consisting of 24 studies comprising altogether 538 patients on the performance of fluorine-18 fluorodeoxyglucose $\left({ }^{18} \mathrm{~F}-\mathrm{FDG}\right) \mathrm{PET}$ or PET/CT in detection of recurrent MTC. The pooled detection rate on a per patient-based analysis was $59 \%$ (range 24-95\%). The authors conclude that ${ }^{18} \mathrm{~F}-\mathrm{FDG}-\mathrm{PET} /(\mathrm{CT})$ is non-optimal in surveillance of MTC since about $40 \%$ of suspected recurrences remain unidentified. Of note, the definition of false negative and true negative imaging results differed. Some considered patients with increased $\mathrm{Ct}$ concentrations and negative ${ }^{18} \mathrm{~F}$-FDG-PET/other imaging as false negative results, while other considered them as true negative. However, a detection rate of $59 \%$ should be related to the corresponding overall detection rates of $\mathrm{CI}$ of about $40 \%$. The current meta-analysis did not present data on whether the ${ }^{18}$ F-FDG-PET studies primarily included patients for whom CI remained negative or not. In clinical practice, ${ }^{18} \mathrm{~F}$-FDG-PET is performed in patients with biochemical proof of recurrence but negative CI. Even a low positive detection rate of ${ }^{18}$ F-FDG-PET in such patients will affect the management of a significant number of patients. In line with this, Skoura et al. [5] reported an overall per-patient sensitivity of $44.1 \%$ in 51 MTC patients with increased Ct 
concentrations and negative CI. The overall sensitivity of ${ }^{18}$ F-FDG-PET was lower (23\%) in patients with MEN2A syndrome.

The meta-analysis by Treglia et al. [4] reported increased detection rates of ${ }^{18} \mathrm{~F}-\mathrm{FDG}-\mathrm{PET} /(\mathrm{CT})$ of $69 \%$ if CEA was $>5 \mathrm{ng} / \mathrm{mL}, 75 \%$ if $\mathrm{Ct}$ was $>1,000 \mathrm{ng} / \mathrm{L}, 76 \%$ if $\mathrm{Ct}$ Dt was $<12$ months and as high as $91 \%$ if CEA Dt was $<24$ months. In line with this, we observed a strong correlation between elevated tumor marker concentrations and ${ }^{18}$ F-FDG PET/CT SUV $\max$ [6]. However, the same trend was observed for fluorine-18 dihydroxyphenylalanine $\left({ }^{18}\right.$ F-DOPA) PET/CT [6]. Skoura et al. [5] reported a sensitivity of $86.7 \%$ for $\mathrm{Ct}>1,000 \mathrm{ng} / \mathrm{L}$ and Ong et al. [7] reported that the sensitivity of ${ }^{18} \mathrm{~F}$-FDG-PET decreases from $78 \%$ for $\mathrm{Ct}>1,000 \mathrm{ng} / \mathrm{L}$, to $20 \%$ for $\mathrm{Ct}<500 \mathrm{ng} / \mathrm{L}$. Many studies have pointed out the importance of tumor marker Dts as compared to single $\mathrm{Ct}$ or CEA concentrations. Koopmans et al. [8] reported that ${ }^{18} \mathrm{~F}-\mathrm{FDG}-\mathrm{PET} / \mathrm{CT}$ seems superior to ${ }^{18} \mathrm{~F}$-DOPA-PET/CT if $\mathrm{Ct} \mathrm{Dt}$ is short ( $<12$ months). Further, detection rate was better with ${ }^{18}$ F-FDG-PET/CT compared to ${ }^{18} \mathrm{~F}$-DOPA-PET/CT in five patients characterized by unstable CEA Dt $(<24$ months) [5]. Exploration of how Dt correlates with the different imaging techniques has further improved the understanding of the seemingly conflicting results of ${ }^{18}$ F-FDG- and ${ }^{18}$ F-DOPA-PET/CT imaging. As differentiated tumors grow slowly, they usually have normal glucose metabolism and are therefore poorly depicted by ${ }^{18} \mathrm{~F}-\mathrm{FDG}-\mathrm{PET} / \mathrm{CT}$. Thus, ${ }^{18} \mathrm{~F}-\mathrm{FDG}-\mathrm{PET} / \mathrm{CT}$ is more optimal for imaging of poorly differentiated MTC than ${ }^{18} \mathrm{~F}$-DOPA-PET/CT.

Since MTC belongs to the group of NETs that are capable of taking up and decarboxylating amine precursors, PET/CT using aminoacid precursor tracers such as ${ }^{18}$ F-DOPA has been proposed to be more specific [6, 8-10]. Importantly, we reported that ${ }^{18}$ F-DOPA-PET/CT was the only method that accurately localized a metastasis in a patient with a $\mathrm{Ct}$ of only $155 \mathrm{pg} / \mathrm{mL}(44.8 \mathrm{pmol} / \mathrm{L})$ in the neck/mediastinal region, with a low $\mathrm{Ki} 67 \%$ tumor proliferation index of $5 \%$ [6]. ${ }^{18}$ F-DOPA-PET/CT seems to be of particular value for detection of occult recurrent MTC characterized by a low proliferation index.

The meta-analysis by Treglia et al. [3] highlights some of the difficulties of ${ }^{18}$ F-FDG-PET imaging with regards to indolent course of MTC and small tumor lesions, which makes the detection rate far from optimal. Giraudet et al. [11] reported that when compared to CT and MRI, ${ }^{18}$ F-FDG-PET seemed to be inferior in detecting cervical and mediastinal lymph node metastases. It is important to recognize the pitfalls of ${ }^{18} \mathrm{~F}$-FDG-PET in the imaging of different NETs.

Today, new tracers are continuously developed for the imaging of NETs. Based on the high-density expression of somatostatin receptors in NETs, somatostatin receptor PET imaging is currently the method of choice for staging of NETs other than MTC [12]. Data on the performance of

${ }^{68} \mathrm{Ga}$-somatostatin imaging in MTC is so far scarce. The study by Koukouraki et al. [13] included one MTC patient with a relatively low SUV. Two retrospective series in recurrent MTC did not indicate superiority of ${ }^{68} \mathrm{Ga}$-somatostatin analogue PET/CT compared to ${ }^{18} \mathrm{~F}-\mathrm{FDG}-$ $\mathrm{PET} / \mathrm{CT}$ and/or ${ }^{18} \mathrm{~F}-\mathrm{DOPA}-\mathrm{PET} / \mathrm{CT}[9,14]$. It is possible that ${ }^{68} \mathrm{Ga}$-somatostatin analogue PET/CT imaging will be less important in MTC compared to other NETs, as MTC tumor cells have a low expression of somatostatin receptors [15].

Recent studies have shed light on the role of ${ }^{18}$ F-FDG$\mathrm{PET} / \mathrm{CT}$ and other PET/CT imaging in recurrent MTC. CI is still recommended as first-line imaging. In the work-up of persistent or recurrent MTC, more emphasize should be put on defining the subtype of the disease to choose the best possible second-line imaging when CI fails and thereby enable the best possible treatment for individual patients. Characteristics of aggressive disease include a high MTC tumor proliferation index ( $\mathrm{Ki} 67 \%$ ), increased CEA rather than $\mathrm{Ct}$ concentrations, $\mathrm{Ct}>1,000 \mathrm{pg} / \mathrm{mL}$ and/or short tumor Dts. ${ }^{18} \mathrm{~F}-\mathrm{FDG}-\mathrm{PET} / \mathrm{CT}$ is the best second-line imaging of such patients, who also are candidates for targeted systemic therapies such as vandetinib. In patients with occult disease, low MTC tumor Ki $67 \%$, only moderately increased $\mathrm{Ct}$ and negative CI, ${ }^{18} \mathrm{~F}$-DOPA-PET/CT is the method of choice. Such patients are candidates for surgical removal or other local treatments of metastases.

\section{References}

1. E. Kebebew, O.H. Clark, Medullary thyroid cancer. Curr. Treat. Options Oncol. 1, 359-367 (2010)

2. G. Pellegriti, S. Leboulleux, E. Baudin, N. Bellon, C. Scollo, J.P. Travagli, M. Schlumberger, Long-term outcome of medullary thyroid carcinoma in patients with normal postoperative medical imaging. Br. J. Cancer 88, 1537-1542 (2003)

3. M. Sclumberger, L. Bastholt, H. Dralle, B. Jarzab, F. Pacini, J.W.A. Smit, The European Thyroid Association guidelines 2012 European Thyroid Association guidelines for metastatic medullary thyroid cancer. Eur. Thyroid J 1, 5-14 (2012)

4. G. Treglia, M.F. Villani, A. Giordano, V. Rufini, Detection rate of recurrent medullary thyroid carcinoma using fluorine-18 fluorodeoxyglucose positron emission tomography: a meta-analysis. Endocrine (2012). doi:10.1007/s12020-012-9671-6

5. E. Skoura, I.E. Datseris, P. Rondogianni, S. Tsagarakis, M. Tzanela, M. Skilakaki, D. Exarhos, M. Alevizaki, Correlation between calcitonin levels and [(18)F]FDG-PET/CT in the detection of recurrence in patients with sporadic and hereditary medullary thyroid cancer. ISRN Endocrinol (2012). doi: $10.5402 / 2012 / 375231$

6. S. Kauhanen, C. Schalin-Jantti, M. Seppanen, S. Kajander, S. Virtanen, J. Schildt, I. Lisinen, A. Ahonen, I. Heiskanen, M. Vaisanen, J. Arola, P. Korsoff, T. Ebeling, T. Sane, H. Minn, M.J. Valimaki, P. Nuutila, Complementary roles of 18F-DOPA $\mathrm{PET} / \mathrm{CT}$ and 18F-FDG PET/CT in medullary thyroid cancer. J. Nucl. Med. 52, 1855-1863 (2011) 
7. S.C. Ong, H. Schoder, S.G. Patel, I.M. Tabangay-Lim, I. Doddamane, M. Gonen, A.R. Shaha, R.M. Tuttle, J.P. Shah, S.M. Larson, Diagnostic accuracy of 18F-FDG PET in restaging patients with medullary thyroid carcinoma and elevated calcitonin levels. J. Nucl. Med. 48, 501-507 (2007)

8. K.P. Koopmans, J.W. de Groot, J.T. Plukker, E.G. de Vries, I.P. Kema, W.J. Sluiter, P.L. Jager, T.P. Links, 18F-dihydroxyphenylalanine PET in patients with biochemical evidence of medullary thyroid cancer: relation to tumor differentiation. J. Nucl. Med. 49, 524-531 (2008)

9. M. Beheshti, S. Pocher, R. Vali, P. Waldenberger, G. Broinger, M. Nader, S. Kohlfurst, C. Pirich, H. Dralle, W. Langsteger, The value of 18F-DOPA PET-CT in patients with medullary thyroid carcinoma: comparison with 18F-FDG PET-CT. Eur. Radiol. 19, 1425-1434 (2009)

10. G. Treglia, P. Castaldi, M.F. Villani, G. Perotti, C. de Waure, A. Filice, V. Ambrosini, N. Cremonini, M. Santimaria, A. Versari, S. Fanti, A. Giordano, V. Rufini, Comparison of 18F-DOPA, 18F-FDG and 68 Ga-somatostatin analogue PET/CT in patients with recurrent medullary thyroid carcinoma. Eur. J. Nucl. Med. Mol. Imaging 39, 569-580 (2012)

11. A.L. Giraudet, D. Vanel, S. Leboulleux, A. Auperin, C. Dromain, L. Chami, N.N. Tovo, J. Lumbroso, N. Lassau, G. Bonniaud,
D. Hartl, J.P. Travagli, E. Baudin, M. Schlumberger, Imaging medullary thyroid carcinoma with persistent elevated calcitonin levels. J. Clin. Endocrinol. Metab. 92, 4185-4190 (2007)

12. G. Treglia, P. Castaldi, G. Rindi, A. Giordano, V. Rufini, Diagnostic performance of gallium-68 somatostatin receptor PET and $\mathrm{PET} / \mathrm{CT}$ in patients with thoracic and gastroenteropancreatic neuroendocrine tumours: a meta-analysis. Endocrine (2012). doi: 10.1007/s12020-012-9631-1

13. S. Koukouraki, L.G. Strauss, V. Georgoulias, J. Schuhmacher, U. Haberkorn, N. Karkavitsas, A. Dimitrakopoulou-Strauss, Evaluation of the pharmacokinetics of 68 Ga-DOTATOC in patients with metastatic neuroendocrine tumours scheduled for 90Y-DOTATOC therapy. Eur. J. Nucl. Med. Mol. Imaging 33, 460-466 (2006)

14. B.G. Conry, N.D. Papathanasiou, V. Prakash, I. Kayani, M. Caplin, S. Mahmood, J.B. Bomanji, Comparison of (68)Ga-DOTATATE and (18)F-fluorodeoxyglucose PET/CT in the detection of recurrent medullary thyroid carcinoma. Eur. J. Nucl. Med. Mol. Imaging 37, 49-57 (2010)

15. J.C. Reubi, J.C. Schaer, B. Waser, G. Mengod, Expression and localization of somatostatin receptor SSTR1, SSTR2, and SSTR3 messenger RNAs in primary human tumors using in situ hybridization. Cancer Res. 54, 3455-3459 (1994) 